Bio-grafía. Escritos sobre la Biología y su Enseñanza. ISSN 2027-1034

Número Extraordinario. p.p. 55-64

Memorias del Primer encuentro ambiental Universidad, ambiente y sustentabilidad: experiencias y prácticas.

\title{
CIUDADANÍA AMBIENTAL DESDE LAS PRÁCTICAS DE MEDICIÓN EN LA CONSTRUCCIÓN DEL ESPACIO DE REFORESTACIÓN
}

Edwin Alberto Gómez ${ }^{1}$

\section{Resumen}

Como estudiante de la Maestría en Docencia de las Matemáticas de la Universidad Pedagógica Nacional, y profesor de matemáticas de la educación básica (en colegios distritales) permitió el diseñó de un ambiente de aprendizaje en su implementación y análisis, en grado sexto, centrado en promover la participación para la construcción del espacio social de reforestación desde las prácticas sociales de medición para favorecer la visibilidad de objetos dentro de la cotidianidad, que establecen su relevancia acorde con sus intereses (lugar social) y los de su comunidad. Experiencia que permite reconocer las prácticas de medición latentes en el grupo para la reflexión de su realidad.

Al implementar el escenario de aprendizaje y analizar las actividades propuestas, originan en estudiantes de grado sexto aspectos favorables para la construcción del espacio social en particular de la percepción que se tiene sobre la reforestación y su relevancia acorde con sus intereses (lugar social) y los de su comunidad. Así mismo permite reconocer que la práctica de medición latente en este grupo, está dada en la transformación de toda característica, en segmento rectos de magnitud métrica, caso que se observó con regularidad en la discusión de volumen y peso; centrando la descripción de objetos en referencias lineales (unidad metro).

\section{Palabras clave:}

Prácticas de Medición. Ciudadanía Ambiental, Construcción del Espacio Social, Reforestación.

${ }^{1}$ Universidad Pedagógica Nacional. - Estudiante de la Maestría en Docencia de las Matemáticas, Profesor de Educación Básica y Media Secretaría de Educación del Distrito. edwinalbertogomezlindo@gmail.com 
Bio-grafía. Escritos sobre la Biología y su Enseñanza. ISSN 2027-1034

Número Extraordinario. p.p. 55-64

Memorias del Primer encuentro ambiental Universidad, ambiente y sustentabilidad: experiencias y prácticas.

\section{Abstract}

As a student of Masters in Teaching of Mathematics of the National Pedagogical University and as a professor of mathematics in basic education (in district schools) was designed, implemented and analyzed, proposed activities in a learning scenario in grade sixth focused on promoting participation in the construction of social space reforestation from social measurement practices to promote the visibility of objects of everyday life and establish its relevance according to their interest (social place) and their community. Experience that allowed latent recognize measurement practices in this group for the analysis of reality.

By implementing the learning scenario and analyze the proposed activities promoted in sixth grade favorable aspects for the construction of social space reforestation because they make visible objects of daily life and establish its relevance according to their interest (social place) and their community. It also allows to recognize that a practice of measuring latent in this group are given in the transformation of the whole property, at right segment metric magnitude, if regularly observed in the discussion of volume and weight.

\section{Keywords:}

Measuring Practices, Environmental Citizenship, Social Space Construction, Reforestation.

\section{OBJETIVO}

Analizar las potencialidades y dificultades de un ambiente de aprendizaje con referencia a prácticas de medición en la construcción del espacio social de reforestación.

\section{MARCO TEÓRICO}

Para crear y generar espacios vivenciales, en los cuales la educación matemática haga parte de un esfuerzo democrático, es necesario involucrar y redefinir las matemáticas en conexión con el contexto social en el que operan 
Bio-grafía. Escritos sobre la Biología y su Enseñanza. ISSN 2027-1034

Número Extraordinario. p.p. 55-64

Memorias del Primer encuentro ambiental Universidad, ambiente y sustentabilidad: experiencias y prácticas.

y con los fenómenos educativos, para considerar una "transposición didáctica" en la concepción de fenómeno social, del cual surgen las matemáticas escolares (Valero \& Skovsmose, 2012). Estas consideraciones permiten establecer dinámicas de reflexión para la creación de escenarios de trabajo que promuevan la participación social de los involucrados en el proceso de promoción de la ciudadanía desde la educación matemática escolar.

Por otra parte, la educación matemática desde el aula, debe representar formas democráticas de interacción, transformación, deliberación y coflexión, entendida esta última como la reflexión colectiva de transformación basada en la deliberación; por lo tanto, estas dos últimas (deliberación y coflexión) se constituyen en recursos para el aprendizaje de las matemáticas a lograr " $E I$ rompimiento de la neutralidad política demanda una acción deliberada para comprometer la educación matemática con la democracia" (Valero \& Skovsmose, 2012, pág. 23).

La práctica social de medición resulta de la exigencia social para comunicar propiedades de la materia mediante una determinada implementación tecnológica (medida), que dispone agentes productivos, recursos, tiempo y espacio para el aprendizaje y la producción efectiva (formas de ser de los objetos), lo que dará origen a las situaciones de relación de los objetos constituyentes (maneras de estar). La reunión entre áreas de actividad permitirá a su vez la ubicación de los" lugares sociales" para establecer las prácticas de medición (Castro, et al, 1996). Es así que, un pucho adquiere significados diferentes: en un grupo de barqueros será dimensionado como un tabaco corto, mientras que, en la comunidad de las plazas de mercado de Bogotá, será asumida como una unidad de medida para los productos en venta.

Al observar elementos naturales constitutivos de las condiciones medioambientales del espacio natural, como el bosque, los nacimientos de agua, la ubicación geográfica, entre otras, que hacen parte de la localización del colegio, sitio de estudio, se puede establecer no sólo el aula como un espacio social, puesto que el entorno se constituye en un medio relacional de la apropiación de la materialidad por parte de hombres, mujeres y niños de la comunidad, (Castro et al, 1996), lo que hace que se reconozcan las prácticas 
Bio-grafía. Escritos sobre la Biología y su Enseñanza. ISSN 2027-1034

Número Extraordinario. p.p. 55-64

Memorias del Primer encuentro ambiental Universidad, ambiente y sustentabilidad: experiencias y prácticas.

de medición como una actividad social, debido a la necesidad de categorizar, expresar y comunicar las formas de ser y del estar de los objetos intangibles y tangibles, así como el reconocimiento, descripción y conservación del entorno (bosque) de trabajo: el suelo, la basura, las semillas, el clima, entre otros.

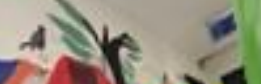

Es la apropiación de la materialidad del bosque natural la que exige restituir el medio ambiente que depende de la creciente necesidad de mejorar la estructura del suelo por los hechos acaecidos en el sector, como deslizamientos constantes que exigen la reubicación de familias, caída de árboles sobre las casas, comercialización del eucalipto y el deterioro de los bosques que inciden a su vez en el deterioro de las fuentes hídricas y acceso al agua para la comunidad, lo que hace necesario integrar de manera dinámica el bosque natural y el territorio con las matemáticas, puesto que supone un espacio de subsistencia al mismo tiempo que un lugar de cohesión o coerción, es decir, un espacio político (Castro, y otros, 1996).

Gracias a la presencia de elementos constitutivos y de condiciones medioambientales particulares del área (entendidas como el espacio de reforestación), el espacio (suelo en el cual se ubican), el bosque, el cual es objeto de reforestación, se convierte en un espacio social donde la relación de los sujetos y el entorno físico y material se constituyen en un factor de apropiación de la materialidad. Así, se genera una dinámica, donde la concepción de dimensión, longitud y unidad (como característica cuantificable de la materialidad) cobra una fuerte relevancia entre los aspectos de espacio natural y territorio, ya que es para los estudiantes su espacio de subsistencia, en donde la cohesión social podrá propiciar espacios políticos de participación, que parten de la necesidad de cuidado de su entorno y que pueden trascender en cambios sociales a futuro (Castro, y otros, 1996).

Así mismo, la interdisciplinariedad identificada en la composición de necesidades en la reforestación de los bosques circundantes, permitiendo a través de las prácticas de medición, reconocer aspectos descripción, reflexión, distribución y control; para establecer, diseñar e implementar instrumentos que apoyen sus intereses, permitiendo encontrar la relación entre las matemáticas, las ciencias y la tecnología, consideradas como aspectos relevantes en el proceso de estimular el aprendizaje significativo, la 
Bio-grafía. Escritos sobre la Biología y su Enseñanza. ISSN 2027-1034

Número Extraordinario. p.p. 55-64

Memorias del Primer encuentro ambiental Universidad, ambiente y sustentabilidad: experiencias y prácticas.

apropiación de saberes y el desarrollo de la reflexión de sujetos, garantizan no sólo la protección del medio ambiente, sino la participación activa y responsable de la comunidad, que determina lo que se ha considerado una práctica socio económica y política de acuerdo a lo establecido en este ambiente de aprendizaje innovador.

\section{4. $t^{2}=$}

La ciudadanía ambiental se interpreta desde la incorporación de la defensa de los ecosistemas, el respeto a la comunidad y sus formas de vida (Gudynas, 2009), para la construcción de "sujetos políticos que activamente participan en la esfera pública en debatir los asuntos de bien común y del bien de la Naturaleza" (Gudynas, 2009, pág. 83), a quienes la experiencia y el reconocimiento de su territorio les permitirá resarcir el impacto generado por la comunidad en los bosques circundantes a la institución educativa, ya que la inadecuada explotación de los recursos, como el establecimiento de canteras, han generado deforestación y deslizamientos de tierra, obligando la reubicación de 14 familias y generando cambios internos en el barrio, lo que ha traído como consecuencia el cierre de vías. Este aspecto, permite fortalecer el proyecto institucional y en particular los objetivos del área de matemáticas, interpretando la realidad y aportando a ella ciudadanos ambientales críticos de sus problemáticas; pero esta acción demanda el repensar las experiencias en el aula. Este espacio debe ser discutido en su complejidad, en su aspecto geográfico, geométrico, temporal, social y emocional. Discutir el espacio teniendo en cuenta los valores y elementos socio-culturales de esta construcción, nos lleva a una perspectiva de conocimiento matemático dinámico, integrado y vinculado a los significados que reelaboran el debate que se establece entre las diversas posibilidades de pensamiento (Lima \& Monteiro, 2009).

La importacia que tiene la restauración ecológica se deriva de la respuesta que la comunidad ha tenido por parte del terreno en el cual habita, que no ha sido otra que la de los deslizamientos y la degradación de los recursos naturales. Esto ha sido consecuencia del mal uso del suelo del sector, lo que ha impactado en la comunidad, cuyo origen se remonta a la explotación de cantera y en la actualidad continúa con la misma actividad, pero con la extracción del eucalipto, lo que ha ocasionado un fuerte deterioro del manto vegetal. Esto ha demandado, por parte de la misma comunidad, considerar la reforestación como una acción que no sólo garantiza la seguridad y estabilidad del terreno, sino también, la existencia del manto vegetal para 
Bio-grafía. Escritos sobre la Biología y su Enseñanza. ISSN 2027-1034

Número Extraordinario. p.p. 55-64

Memorias del Primer encuentro ambiental Universidad, ambiente y sustentabilidad: experiencias y prácticas.

futuras generaciones, en particular esta generación en formación de nuestro colegio. Con lo anteriormente expuesto, es necesariocontemplar las dimensiones que requieren la atención de esta acción (reforestación) por la comunidad y el sector a considerar (Gálvez, 2002).

\section{METODOLOGÍA}

Este estudio se inscribe en la línea de investigación de Diversidad, Educación Matemática y Subjetividades. Se propone relacionar las estructuras sociales, económicas y políticas y su relación con el aula, profundizando la enseñanza y aprendizaje de las matemáticas, según la cultura establecida en la clase. Es importante señalar que esta perspectiva, identifica los supuestos sobre los sujetos del aprendizaje y trasciende la mirada exclusiva de lo cognitivo, ya que se asume el aprendizaje como una acción en la cual la persona toma la decisión de participar (Skovsmose, 1999). La participación depende de la relación que el estudiante establece entre las actividades del aula, su mundo y en particular, sus posibilidades futuras de vida. (Skovsmose, 2005); (Skovsmose et al, 2007); (García, Valero, Camelo, Mancera, 2009). Se afirma entonces que los estudiantes se consideran como seres actuantes, que toman decisiones para participar en el aprendizaje de las matemáticas y en la reflexión de su cotidianidad.

Siendo consecuentes con los referentes expuestos, la metodología que orienta el estudio es la investigación-acción. El esquema general del estudio corresponde a la interacción entre ciclos que se describen como fundamentales para este trabajo. El modelo Kemmis (1989), citado por La Torre (2007), describe los ciclos que se amplían en la permanente reflexión y acción del proceso seguido en el estudio. Cada uno de estos ciclos se desarrolla en cuatro fases que comprenden: la elaboración de un plan de acción, la actuación, la implementación y la reflexión colectiva sobre los resultados obtenidos.

El estudio se realizó en la clase de matemáticas del curso 601, del cual se seleccionó un grupo conformado por ocho estudiantes, un niño y siete niñas. Una de ellas tiene diagnóstico de necesidades educativas especiales (NEE), según su coeficiente intelectual y diagnóstico especializado (psicólogo y psicopedagógico). 
Bio-grafía. Escritos sobre la Biología y su Enseñanza. ISSN 2027-1034

Número Extraordinario. p.p. 55-64

Memorias del Primer encuentro ambiental Universidad, ambiente y sustentabilidad: experiencias y prácticas.

En relación al tipo de tareas realizadas por parte de los estudiantes de grado sexto, se les propuso interpretar las unidades de medida, en longitud, perímetro, área y volumen para establecer proporcionalidades y relacionar las magnitudes seleccionadas, lo que permitió recolectar esta información como instrumento de indagación.

Los referentes dados y las experiencias descritas para la construcción del escenario centrado en las prácticas de medición, asumieron como referencia la construcción del espacio físico de reforestación en el bosque según espacio social de participación. El escenario estuvo constituido por cuatro escenas: Reconocimiento y delimitación del espacio de reforestación, análisis de los suelos, preparación del espacio y reforestación.

\section{CONCLUSIONES}

Luego de varias sesiones de trabajo con el grupo, son diversas y variadas las conclusiones a las que se pudo llegar una vez se logró el análisis de las potencialidades y dificultades en la participación democrática de los estudiantes en este ambiente de aprendizaje, con referencia a prácticas de medición en la construcción del espacio social de reforestación.

Es posible afirmar que se estableció una relación evidente entre las matemáticas y la participación democrática de los estudiantes en ambientes de aprendizaje con referencia a prácticas de medición en la construcción del espacio de reforestación, debido a que la primera fue una herramienta primordial en la construcción y aplicación de conceptos que ayudaron con la tarea propuesta (Skovsmose, 2000).

La construcción y montaje del escenario de aprendizaje no se centró exclusivamente en el contenido matemático, sino que, por el contrario, se puso de relieve la cultura de los estudiantes con relación a su participación democrática en los ambientes de aprendizaje con referencia a prácticas de medición y la construcción del espacio de reforestación, ya que el grupo estaba familiarizado con la zona en donde se trabajó, factor que facilitó en alguna medida el objetivo de la investigación.

El escenario permitió observar la existencia de otro tipo de actividades, que podían llegar a ser más cercanas a los estudiantes, en espacios diferentes al 
Bio-grafía. Escritos sobre la Biología y su Enseñanza. ISSN 2027-1034

Número Extraordinario. p.p. 55-64

Memorias del Primer encuentro ambiental Universidad, ambiente y sustentabilidad: experiencias y prácticas.

aula de clase, elaborando preguntas abiertas que generaron discusiones y reflexiones en un compromiso con la comunidad. En nuestro quehacer como docentes, se involucraron actividades grupales, con multiplicidad de respuestas que motivaron el diálogo.

La búsqueda de nuevas formas de comunicación en la clase de matemáticas, en donde el discurso autoritario no fuera la única opción, permitió descubrir que el diálogo, el debate argumentado y la participación democrática de los estudiantes, podían modificar las prácticas y procedimientos pedagógicos tradicionales, entre ellos, las formas de comunicación estudiante-estudiante y estudiante-profesor. Para ello, fue necesario iniciar un cambio en los esquemas mentales, donde los estudiantes se animarán más a participar del proceso de elaboración de un ambiente de aprendizaje con referencia a prácticas de medición en la construcción del espacio de reforestación.

El aprendizaje de los estudiantes en el nuevo escenario de medición y reforestación, significó una mayor interacción de la comunidad con su entorno. Al tomar conciencia de la importancia que para el desarrollo de su barrio tuvo la actividad de reforestación, los jóvenes generaron en los adultos nuevas acciones que les han permitido observar los cerros como algo más importante que un espacio de explotación de la vegetación. Son ahora conscientes de que se trata de una herencia material y casi espiritual para la actual generación y para las generaciones venideras. 
Bio-grafía. Escritos sobre la Biología y su Enseñanza. ISSN 2027-1034

Número Extraordinario. p.p. 55-64

Memorias del Primer encuentro ambiental Universidad, ambiente y sustentabilidad: experiencias y prácticas.

\section{REFERENTES BIBLIOGRAFÍCOS}

Campbell, N. R. (1994). Medición. En J. R. Newman, Sigma El mundo de las matemáticas (págs. 186 -201). Barcelona: Ediciones Grijalbo, S.A.

Carrillo, J., Climent, N., Gorgorió, N., Prat, M., \& Rojas, F. (2008). Análisis de Secuencias de Aprendizaje Matemático desde la Perspectiva de la Gestión de la Participación. Enseñanza de las Ciencias, pp. 67-76.

Castro, P., Chapman, R., Gui Suriñach, S., Lull, V., Micó Pérez, R., Rihuete, C., y otros. (1996). Teoría de las Prácticas Sociales. Complutum Extra, 3548.

Chamorro Plaza, M. d., \& Belmonte Gómez, J. M. (1991). El Problema de la medida. Vallehermosa Madrid: Editorial Síntesis S.A.

Gálvez, J. (2002). La restauración Ecológica: concepto y aplicación. Guatemala: Universidad Rafael Landivar Facultad de Ciencias Ambientales y Agrícolas.

García, G., Valero, P. x., Salazar, C., Mancera, G., Camelo, F. J., \& Romero, J. H. (2013). Escenarios y Ambientes educativos de aprendizaje de las matemáticas. Constitución de subjetividades en educación matemática elemental. En G. García, p. x. Dueñas, C. S. Amaya, G. M. Ortiz, F. J. Bustos, \& J. H. Rey, Proceso de inclusión exclusión Subjetividades en Educación Matemática (págs. 45- 76). Bogotá: Javergraf.

Godino, J., Batanero, C., \& Roa, R. (2002). Medida de Magnitudes y su Didáctica para Maestros. Granada: Publicación realizada en el marco del Proyecto de Investigación y Desarrollo del Ministerio de Ciencia y Tecnología.

Gudynas, E. (2009). Ciudadanía ambiental y meta-ciudadanías ecológicas. Revisión y alternativas en América Latina. En J. Reyes, \& E. Castro, Urgencias y utopía a la crisis de civilización (págs. 58 - 101). Guadalajara.

Lima, M. J., \& Monteiro, A. (2009). Práticas Sociais de Localização e Mapeamento: Bolema, 1 -28.

MEN. (2006). Plan decenal de educación 2006-2016. Recuperado el 13 de agosto de 2013, de http://www.plandecenal.edu.co/html/1726/w3-article166057.html 
Bio-grafía. Escritos sobre la Biología y su Enseñanza. ISSN 2027-1034

Número Extraordinario. p.p. 55-64

Memorias del Primer encuentro ambiental Universidad, ambiente y sustentabilidad: experiencias y prácticas.

Monteiro, A., \& Rodrigues, J. M. (2011). Prácticas sociales y organización curricular: cuestiones y desafíos. Revista Educación y Pedagogía, 37 - 46.

Pedro V Castro, R. E. (1996). Teoría de las Prácticas Sociales. 35 - 48.

Pereira, M. G. (2011). La Construcción de Espacio Escolar y La Justicia Social. Revista Geográfica de América Central, 1-21.

Valero, P., \& Skovsmose, O. (2012). Educación Matemática Crítica Una Visión Sociopolítica del Aprendizaje y la Enseñanza de las Matemáticas. Colombia: Ediciones Uniandes.

Vásquez Yanes, C., Batis Muños, A. I., \& Alcocer, M. I. (2004). Árboles y Arbustos Nativos Potencialmente Valiosos para la Restauración Ecológica y la Reforestación. México: CONABIO. 